\title{
LHCb upgrade plans
}

\author{
F. Muheim ${ }^{\mathrm{a}}$ \\ On behalf of the LHCb Collaboration

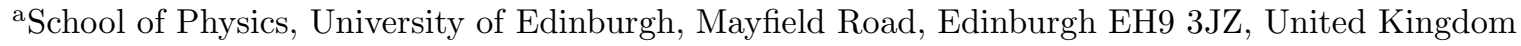

The LHCb experiment will operate for about five years at a luminosity of $2 \times 10^{32} \mathrm{~cm}^{-2} \mathrm{~s}^{-1}$ and plans are to accumulate a data sample of $\sim 10 \mathrm{fb}^{-1}$. Here we present the physics programme and detector design for a future high luminosity phase of the LHCb experiment. An upgraded LHCb experiment would operate at ten times the design luminosity, i.e. at $\sim 2 \times 10^{33} \mathrm{~cm}^{-2} \mathrm{~s}^{-1}$ and aims to collect a data sample of $\sim 100 \mathrm{fb}^{-1}$ over five years. This programme would allow the probe of new physics at an unprecedented level. Key measurements include the $B_{s}^{0}$ mixing phase $\phi_{s}$ in $B_{s}^{0} \rightarrow J / \psi \phi$ and $B_{s}^{0} \rightarrow \phi \phi$ decays with a significant sensitivity to the small Standard Model prediction and a very precise measurement of the CKM angle $\gamma$ in tree diagram decays. Initial studies of the modified LHCb trigger and detectors are presented. The upgraded LHCb experiment can run with or without an LHC luminosity upgrade.

\section{INTRODUCTION}

The Standard Model (SM) is a very successful effective theory. Over the last few years it has emerged that the CKM mechanism is likely the dominant mechanism and new physics (NP) beyond the SM would appear as corrections. If NP were observed at the LHC its flavour sector must be studied. Here we discuss the plans for an upgraded $\mathrm{LHCb}$ experiment. In Section 2 we present the $\mathrm{LHCb}$ experiment and physics programme, and motivate running at higher luminosities. The physics reach of a $100 \mathrm{fb}^{-1}$ data sample is discussed in Section 3 for selected key measurements: the $B_{s}^{0}$ mixing phase $\phi_{s}$ from the tree decay $B_{s}^{0} \rightarrow J / \psi \phi$, the $b \rightarrow s$ penguin decay $B_{s}^{0} \rightarrow \phi \phi$, as well as the CKM angle $\gamma$. In Section 4 we present the plans for upgrading the $\mathrm{LHCb}$ trigger and detectors. The report ends with conclusions.

\section{THE LHCb PHYSICS PROGRAMME}

\subsection{The LHCb experiment}

The goal of the $\mathrm{LHCb}$ experiment is to perform precise measurements of $\mathrm{CP}$ violation and rare decays of $B$ mesons. The $\mathrm{LHCb}$ detector is a forward arm spectrometer which is equipped with state-of-the-art vertexing capabilities, and is de- signed to trigger efficiently on muons, electrons and photons as well as hadronic final states. Two Ring Imaging Cherenkov ( $\mathrm{RICH}$ ) detectors allow excellent charged particle identification. The trigger and flavour tagging of $\mathrm{LHCb}$ are discussed elsewhere in these proceedings 12 . The $\mathrm{LHCb}$ experiment will start taking data in 2007 at the Large Hadron Collider (LHC) at CERN. More details about the current status of $\mathrm{LHCb}$ and its first year data-taking plans are described in [34].

\section{2. $\mathrm{LHCb}$ - the first five years}

The $\mathrm{LHCb}$ experiment is designed to operate at a luminosity $\mathcal{L} \sim 2 \times 10^{32} \mathrm{~cm}^{-2} \mathrm{~s}^{-1}$. This choice maximises the number of bunch crossings with exactly one $p p$ interaction and reduces the radiation damage to the vertex detector. This luminosity is a factor of $\sim 50$ below the LHC design value which is achievable by using a tunable amplitude function, $\beta^{*}$, at the $\mathrm{LHCb}$ interaction point. Therefore this luminosity will very likely be reached during the first LHC physics run. In a nominal year of $10^{7} \mathrm{~s}$, a data sample of $2 \mathrm{fb}^{-1}$ will be collected by $\mathrm{LHCb}$.

The large cross section of $500 \mu \mathrm{b}$ for the production of $b \bar{b}$-quark pairs at the LHC will allow $\mathrm{LHCb}$ to collect much larger data samples of selected $B$ meson decays than previously available. 
Table 1

Expected signal yields $S$, signal to background ratios $B / S$ and sensitivities for $2 \mathrm{fb}^{-1}$ of data. The yields also include charge conjugate modes, which are implied throughout. The parameters are defined in the text; $C_{7}^{\text {eff }} / C_{9}^{\text {eff }}$ is the ratio of Wilson coefficients and $A_{C P}$ is the asymmetry in direct CP violation.

\begin{tabular}{clccc}
\hline & Decay & Yield $S$ & $B / S$ & Precision \\
\hline$\gamma$ & $B_{s}^{0} \rightarrow D_{s}^{\mp} K^{ \pm}$ & $5.4 \mathrm{k}$ & $<1.0$ & $\sigma(\gamma) \sim 14^{\circ}$ \\
& $B^{0} \rightarrow \pi^{+} \pi^{-}$ & $36 \mathrm{k}$ & 0.46 & $\sigma(\gamma) \sim 4^{\circ}$ \\
& $B_{s}^{0} \rightarrow K^{+} K^{-}$ & $36 \mathrm{k}$ & $<0.06$ & \\
& $B^{0} \rightarrow D^{0}\left(K^{-} \pi^{+}, K^{+} \pi^{-}\right) K^{* 0}$ & $3.4 \mathrm{k}, 0.5 \mathrm{k}$ & $<0.3,<1.7$ & $\sigma(\gamma) \sim 7^{\circ}-10^{\circ}$ \\
& $B^{0} \rightarrow D^{0}\left(K^{+} K^{-}, \pi^{+} \pi^{-}\right) K^{* 0}$ & $0.5 \mathrm{k}$ & $<1.4$ & \\
& $B^{-} \rightarrow D^{0}\left(K^{-} \pi^{+}, K^{+} \pi^{-}\right) K^{-}$ & $56 \mathrm{k}, 710$ & $0.6,1.5-4.3$ & $\sigma(\gamma) \sim 5^{\circ}-15^{\circ}$ \\
& $B^{-} \rightarrow D^{0}\left(K^{+} K^{-} / \pi^{+} \pi^{-}\right) K^{-}$ & $7.6 \mathrm{k}$ & 1.0 & \\
& $B^{-} \rightarrow D^{0}\left(K_{S}^{0} \pi^{+} \pi^{-}\right) K^{-}$ & $1.5-5 \mathrm{k}$ & $<0.7-2.3$ & $\sigma(\gamma) \sim 8^{\circ}-16^{\circ}$ \\
\hline$\alpha$ & $B^{0} \rightarrow \pi^{+} \pi^{-} \pi^{0}$ & $14 \mathrm{k}$ & $<0.8$ & $\sigma(\alpha) \sim 10^{\circ}$ \\
& $B^{+, 0} \rightarrow \rho^{+} \rho^{0}, \rho^{+} \rho^{-}, \rho^{0} \rho^{0}$ & $9 \mathrm{k}, 2 \mathrm{k}, 1 \mathrm{k}$ & $1,<5,<4$ & \\
\hline$\beta$ & $B^{0} \rightarrow J / \psi K_{S}^{0}$ & $216 \mathrm{k}$ & 0.8 & $\sigma(\sin 2 \beta) \sim 0.022$ \\
\hline$\Delta m_{s}$ & $B_{s}^{0} \rightarrow D_{s}^{-} \pi^{+}$ & $80 \mathrm{k}$ & 0.3 & $\sigma\left(\Delta m_{s}\right) \sim 0.01 \mathrm{ps}^{-1}$ \\
$\phi_{s}$ & $B_{s}^{0} \rightarrow J / \psi \phi$ & $131 \mathrm{k}$ & 0.12 & $\sigma\left(\phi_{s}\right) \sim 0.023 \mathrm{rad}$ \\
\hline Rare & $B_{s}^{0} \rightarrow \mu^{+} \mu^{-}$ & 17 & $<5.7$ & \\
Decays & $B^{0} \rightarrow K^{* 0} \mu^{+} \mu^{-}$ & $4.4 \mathrm{k}$ & $<2.6$ & $\sigma\left(C_{7}^{\text {eff }} / C_{9}^{\text {eff }}\right) \sim 0.13$ \\
& $B^{0} \rightarrow K^{* 0} \gamma$ & $35 \mathrm{k}$ & $<0.7$ & $\sigma\left(A_{C P}\right) \sim 0.01$ \\
& $B_{s}^{0} \rightarrow \phi \gamma$ & $9.3 \mathrm{k}$ & $<2.4$ & \\
\hline & & &
\end{tabular}

The sensitivities of these data-sets to $\mathrm{CP}$ violating asymmetries and to other physics observables have been studied with simulated events. In Table 1 we present a selection of signal yields $S$, background to signal ratios $B / S$ and precisions on CKM angles $\gamma, \alpha, \beta$ and $\phi_{s}$ and other observables. Detailed presentations on many of the channels are presented elsewhere in these proceedings $5667789[10[11]$.

The plan is to operate the $\mathrm{LHCb}$ experiment for five years at the design luminosity and to collect a data sample of 6 to $10 \mathrm{fb}^{-1}$. A major goal is to exploit the physics in the $B_{s}$ system. This includes the observation of $\mathrm{CP}$ violation in $B_{s}$ mesons and precision measurements of the mass difference between the $B_{s}^{0}$ mass eigenstates, $\Delta m_{s}$, and the lifetime difference $\Delta \Gamma_{s}$. Other main aims are to improve the error on the CKM angle $\gamma$ by a factor of five, to probe NP in rare $B$ meson decays with electroweak, radiative and hadronic penguin modes, and to make the first observation of the very rare decay $B_{s}^{0} \rightarrow \mu^{+} \mu^{-}$.

\subsection{LHCb at higher luminosity}

After the first five years of operation, the precision of many LHCb physics results will remain limited by the statistical error of the collected data. The following questions arise: is $\mathrm{LHCb}$ exploiting the full potential for $B$ physics at hadron colliders and is there a science case for collecting even larger data samples? Note that LHCb is the only dedicated heavy flavour experiment approved to run after 2010. In the remainder of this report we will try to answer these questions.

The LHCb experiment has commenced studying the feasibility of upgrading the detector such that it can operate at a luminosity $\mathcal{L} \sim 2 \times$ $10^{33} \mathrm{~cm}^{-2} \mathrm{~s}^{-1}$, which is ten times larger than the design luminosity. This upgrade would allow $\mathrm{LHCb}$ to collect a data sample of about $100 \mathrm{fb}^{-1}$ during five years of running. This increased luminosity is achievable by decreasing $\beta^{*}$ at the $\mathrm{LHCb}$ interaction point. It does not require a LHC luminosity upgrade (Super-LHC) as the LHC design luminosity is $10^{34} \mathrm{~cm}^{-2} \mathrm{~s}^{-1}$ although it could op- 
erate at Super-LHC. Thus an upgrade of $\mathrm{LHCb}$ could be implemented as early as 2013. The number of interactions per beam crossing will increase to $n \sim 4$ which will require improvements to the LHCb sub-detectors and trigger.

\section{LHCb PHYSICS REACH WITH A $100 \mathrm{fb}^{-1}$ DATA SAMPLE}

\subsection{Weak mixing phase $\phi_{s}$}

Flavour-changing neutral currents (FCNCs) are particularly sensitive to NP. New particles appear virtually in loop diagrams which lead to deviations from $\mathrm{SM}$ predictions. The interference in $B_{s}^{0}$ mixing and decay to $\mathrm{CP}$ eigenstates is sensitive to the $\mathrm{CP}$ violating weak mixing phase $\phi_{s}$. This can be studied in the time-dependent asymmetry of flavour-tagged $B_{s}^{0} \rightarrow J / \psi \phi$ decays. The SM prediction for $\phi_{s}$ is very small: $\phi_{s}=-2 \chi=$ $-2 \lambda^{2} \eta \approx-0.035 \mathrm{rad}$ where $\lambda$ and $\eta$ are the usual Wolfenstein parameters of the CKM matrix [12]. Hence $\phi_{s}$ is a very sensitive probe of NP and will provide a stringent test for Non-Minimal Flavour Violation (NMFV) [13.

Currently the only direct $\phi_{s}$ result is from D0 who measure $\phi_{s}=-0.79 \pm 0.56_{-0.01}^{+0.14} \mathrm{rad}$ in the untagged decay time spectrum of $B_{s}^{0} \rightarrow J / \psi \phi$ decays 14. Using a full simulation, the $\mathrm{LHCb}$ experiment expects to collect $131 \mathrm{k} B_{s}^{0} \rightarrow J / \psi \phi$ decays with a $2 \mathrm{fb}^{-1}$ data sample [15]. The expected precision on $\phi_{s}$ is estimated with many toy Monte Carlo experiments based on the yields and resolutions obtained in the full simulation. We obtain $\sigma\left(\phi_{s}\right) \approx 0.023 \mathrm{rad}$ where the $B_{s}^{0}$ mass difference has been set to $\Delta m_{s}=20 \mathrm{ps}^{-1}$. New physics in $B_{s}^{0}$ mixing can be parameterised as $\Delta m_{s}^{N P}=\left(1+h_{s} e^{2 i \sigma_{s}}\right) \Delta m_{s}^{S M}$ where the SM phase of $\Delta m_{s}^{S M}$ is equal to $-2 \chi$ and $h_{s}$ and $\sigma_{s}$ are, respectively, the amplitude and the phase of the NP contribution. In Fig. 1 we show the expected sensitivity for $h_{s}$ and $\sigma_{s}$ for LHCb with $2 \mathrm{fb}^{-1}$ of data [16. With five years of data, $\mathrm{LHCb}$ expects to achieve a precision of $\sigma\left(\phi_{s}\right) \sim$ $0.010 \mathrm{rad}$ which corresponds to about a $3 \sigma$ significance for the SM value of $\phi_{s}$. This precision is expected to be statistically limited, with systematic errors likely to be much smaller. The precision of the SM prediction for $\phi_{s}$ is better than $0.01 \mathrm{rad}$.

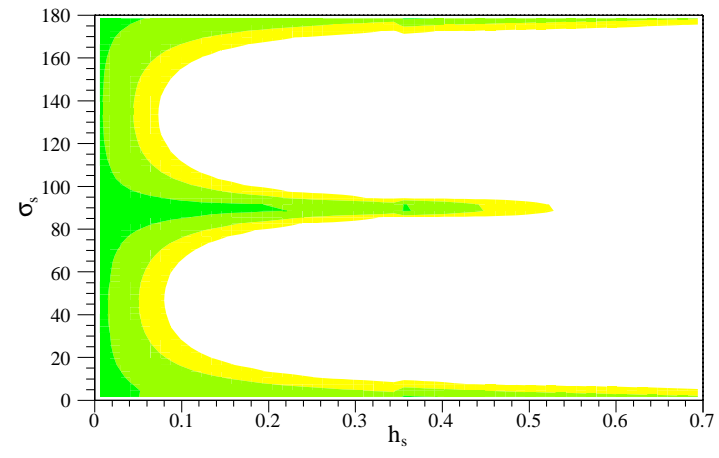

Figure 1. The allowed range for $h_{s}$ and $\sigma_{s}$ with 2 $\mathrm{fb}^{-1}$ of LHCb data. The dark, medium, and light shaded areas have confidence levels $>0.90,0.32$, and 0.05 , respectively, taken from [16].

A significantly larger data-set would allow $\mathrm{LHCb}$ to probe new physics in $B$ mesons at an unprecedented level of a few percent. Here we present estimates for sensitivities with a $100 \mathrm{fb}^{-1}$ data sample which are based on scaling with luminosity. Potential trigger efficiency improvements are not included. While these estimates have large uncertainties, these are extremely useful to motivate simulation studies with the aim to improve or at least maintain trigger and selection efficiencies at ten times higher luminosities. An upgrade of LHCb has the potential to measure the SM value of $\phi_{s}$ with $\sim 10 \sigma$ precision $\left(\sigma\left(\phi_{s}\right) \sim 0.003 \mathrm{rad}\right)$ in $B_{s}^{0} \rightarrow J / \psi \phi$ decays.

\section{2. $b \rightarrow s$ transitions in $B_{s}^{0} \rightarrow \phi \phi$}

New physics can be probed by studying FCNCs in hadronic $b \rightarrow s$ transitions. One approach is to compare the time-dependent $\mathrm{CP}$ asymmetry in a hadronic penguin loop decay, where unknown massive particles could enter with a decay based on a tree diagram which generally is insensitive to NP and which has the same weak phase. The B-factories measure the CP asymmetry $\sin 2 \beta_{\text {eff }}$ in the penguin decay $B^{0} \rightarrow \phi K_{S}^{0}$. A value for $\sin 2 \beta_{\text {eff }}$ which is different from $\sin 2 \beta$ measured in $B^{0} \rightarrow J / \psi K_{S}^{0}$ would signal physics beyond the SM. Within the current available precision, all 
$\sin 2 \beta_{\text {eff }}$ measurements are in reasonable agreement with the SM, but all central values are lower than expected. A naive average indicates a $2.6 \sigma$ discrepancy [17.

The above approach can also be applied to $B_{s}^{0}$ mesons and this will be exploited by LHCb. Within the SM the weak mixing phase $\phi_{s}$ is expected to be almost the same when comparing the time-dependent $\mathrm{CP}$ asymmetry of the hadronic penguin decay $B_{s}^{0} \rightarrow \phi \phi$ with the tree decay $B_{s}^{0} \rightarrow J / \psi \phi$. Due to a cancellation of the $B_{s}$ mixing and decay phase, the SM prediction for $\phi_{s}(\phi \phi)$ is actually very close to zero [18]. Thus any measurement of $\phi_{s}(\phi \phi) \neq 0$ is a clear signal for NMFV. LHCb expects to collect $1.2 \mathrm{k}$ $B_{s}^{0} \rightarrow \phi \phi$ decays in $2 \mathrm{fb}^{-1}$ of data 19 . By comparing with the $B_{s}^{0} \rightarrow J / \psi \phi$ yield we estimate a sensitivity of $\sigma\left(\phi_{s}(\phi \phi)\right) \sim 0.14 \mathrm{rad}$ for $10 \mathrm{fb}^{-1}$ of data. This $\sigma\left(\phi_{s}(\phi \phi)\right)$ precision is statistically limited. Scaling the sensitivity up to a data sample of $100 \mathrm{fb}^{-1}$ we estimate a precision of $\sigma\left(\phi_{s}(\phi \phi)\right) \sim 0.04 \mathrm{rad}$. This sensitivity presents a very precise and exciting NP probe. A first-level detached vertex trigger is required to reconstruct this hadronic decay mode. A new LHCb simulation study on $\sigma\left(\phi_{s}(\phi \phi)\right)$ can be found in [20].

\subsection{CKM angle $\gamma$ from $B \rightarrow D K$ and $B_{s}^{0} \rightarrow D_{s}^{\mp} K^{ \pm}$}

$\mathrm{LHCb}$ will perform direct measurements of the CKM angle $\gamma$ using two interfering tree processes in neutral and charged $B \rightarrow D K$ decays. The interference arises due to decays which are common to $D^{0}$ and $\bar{D}^{0}$ mesons such as $D^{0}\left(\bar{D}^{0}\right) \rightarrow$ $K_{S}^{0} \pi^{+} \pi^{-}$(Dalitz decay 21] ) and $D^{0}\left(\bar{D}^{0}\right) \rightarrow$ $K^{\mp} \pi^{ \pm}, K^{+} K^{-}$(ADS and GLW 22[23]). The current combined direct $\gamma$ measurements at the $B$ factories have large errors of about $30^{\circ}$ [17.

The expected LHCb $\gamma$ sensitivities for $2 \mathrm{fb}^{-1}$ of data have been estimated for several of the above modes [6]; we obtain $\sigma(\gamma) \sim 7^{\circ}-15^{\circ}$. In addition, LHCb will use the decay $B_{s}^{0} \rightarrow D_{s}^{\mp} K^{ \pm}$to measure $\gamma$ with $\sigma(\gamma) \sim 14^{\circ}$ in $2 \mathrm{fb}^{-1}$. By combining these measurements, $\mathrm{LHCb}$ will achieve a precision of $\sigma(\gamma) \sim 5^{\circ}$ in $2 \mathrm{fb}^{-1}$. The theoretical error on the SM prediction is very small. Note that some of the $\gamma$ measurements will have a correlated systematic error, for example there is a

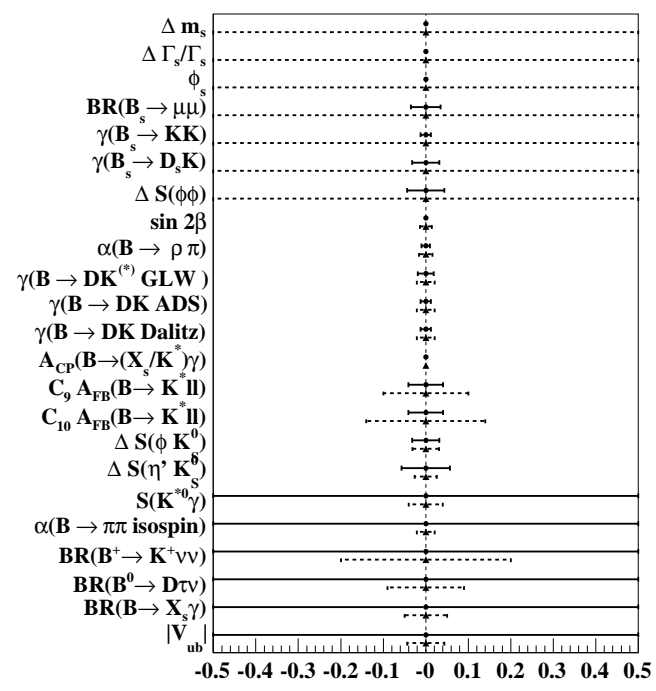

Figure 2. Expected sensitivities for LHCb (solid lines) with $100 \mathrm{fb}^{-1}$ compared to an $e^{+} e^{-}$Super$B$ factory (dashed lines) with a data sample of $50 \mathrm{ab}^{-1}$ 24]. Note that $\Delta S(\phi \phi)=\phi_{s}(\phi \phi)-$ $\phi_{s}(J / \psi \phi), \Delta S\left(\phi K_{S}^{0}, \eta^{\prime} K_{S}^{0}\right)=\sin 2 \beta_{e f f}-\sin 2 \beta$ and $S\left(K^{* 0} \gamma\right)=\sin 2 \beta_{\text {eff }}$.

Dalitz model dependence. We take this into account when scaling to a $100 \mathrm{fb}^{-1}$ data sample and estimate that a precision of $\sigma(\gamma) \sim 1^{\circ}$ should be achievable. This would allow a very precise determination of the apex of the unitarity triangle.

\subsection{Summary and comparison with the Super-B Factory}

We presented a selection of key measurements that could be performed with an upgrade of the LHCb experiment, namely precision measurements of the weak mixing phase $\phi_{s}$ in tree decays and $b \rightarrow s$ transitions, and of the CKM angle $\gamma$. Many more measurements would also benefit from a ten-fold increase in statistics. With $100 \mathrm{fb}^{-1}$ of data, $\mathrm{LHCb}$ would be able measure $\Delta S\left(\phi K_{S}^{0}\right)=\sin 2 \beta_{\text {eff }}-\sin 2 \beta$ in $B^{0} \rightarrow \phi K_{S}^{0}$ with an estimated precision of $\sigma\left(\Delta S\left(\phi K_{S}^{0}\right)\right) \sim 0.04$. 
An estimated yield of $44 \mathrm{k} B^{0} \rightarrow K^{* 0} \mu^{+} \mu^{-}$events per year would allow probing right-handed currents by analysing forward-backward asymmetries $\left(A_{F B}\right)$ and transversity angles. In addition, the LHCb upgrade would allow the probeing of $\mathrm{NP}$ in charm decays and to study lepton flavour violation in $\tau$ decays.

In Fig. 2 we present a comparison of the expected sensitivities for the LHCb upgrade, scaled from 10 to $100 \mathrm{fb}^{-1}$, with sensitivities at a SuperB factory based on a $50 \mathrm{ab}^{-1}$ data sample [24]. Both approaches have comparable sensitivities for many measurements such as $\gamma$ and $\sin 2 \beta_{\text {eff }}$. $\mathrm{LHCb}$ will be limited in reconstructing decays with (more than one) neutral final state particles (photon, $\pi^{0}$ or $\eta$ ). However only an upgrade of the $\mathrm{LHCb}$ experiment will allow an ultimate exploitation of $B_{s}^{0}$ meson decays. While these estimates need to be confirmed with simulations at high luminosities, an excellent science case can be written for an LHCb upgrade.

\section{LHCb DETECTOR AND TRIGGER UPGRADE}

\subsection{Detector and trigger plans}

The trigger for the $\mathrm{LHCb}$ experiment comprises a first level trigger (L0) implemented in hardware and a Higher Level Trigger (HLT) running on a large CPU farm. The L0 trigger operates at $40 \mathrm{MHz}$ and selects high transverse momentum objects in the electromagnetic and hadronic calorimeters ( $e, \gamma$, charged hadrons) and muons in the muon system $(\mu, \mu \mu)$. Pile-up events are vetoed. The output rate is limited to $1.1 \mathrm{MHz}$ with $4 \mu s$ latency. Simulations show that the L0 muon trigger efficiency for reconstructable events is around $90 \%$ and that the output rate scales with luminosity up to $5 \times 10^{32} \mathrm{~cm}^{-2} \mathrm{~s}^{-1}$. At the design luminosity, the L0 hadron trigger efficiencies are about $40 \%$, and the output rate increases only slightly with luminosity. The vertex detector (VELO) sensors [19] undergo radiation damage and it is expected that these will need to be replaced when 6 to $8 \mathrm{fb}^{-1}$ of luminosity has been collected. This illustrates that the existing trigger and at least some of the sub-detector systems will not allow operating the $\mathrm{LHCb}$ experiment at ten times the design luminosity.

We have commenced studies which investigate how to upgrade the $\mathrm{LHCb}$ detector and triggers such that the experiment can operate at luminosities $\mathcal{L} \sim 2 \times 10^{33} \mathrm{~cm}^{-2} \mathrm{~s}^{-1}$. Two scenarios are under consideration. A step-by-step approach would foresee to replace the VELO sensors with improved radiation-hard detectors which will be read out at $40 \mathrm{MHz}$. This would allow the addition of a first-level detached vertex trigger at L0. The Trigger Tracker in front of the LHCb dipole magnet could also be included. This trigger could be implemented in FPGAs, and it would need to be very fast and run within the L0 latency time. Other LHCb detector systems will also need to be upgraded when increasing the luminosity, either due to unacceptably large occupancies or radiation effects. We are currently studying the luminosity limit of each $\mathrm{LHCb}$ sub-detector system. Under discussion is the replacement of the central region of the RICH 1 photon detectors, an increase (decrease) of the Inner (Outer) Tracker area, and a replacement of the inner region of the electromagnetic calorimeter.

Another approach would be to change the readout of all LHCb sub-detectors to $40 \mathrm{MHz}$. This has clear advantages as it would allow the implementation of a L0 displaced vertex trigger in a CPU farm. In fact all trigger decisions would be software-based which allows flexibility. However this approach requires a redesign of the frontend electronics which has implications for all subdetector systems. Besides the VELO, all silicon sensors of the Trigger and Inner Tracker and the RICH photon detectors would need to be replaced. The R\&D for the new front-end electronics will be able to profit from the developments of ATLAS and CMS for Super-LHC luminosities.

\subsection{Initial studies}

R\&D efforts have started on technologies for radiation-hard vertex detectors that will be able to operate in the radiation environments of the LHC and LHCb upgrade. The detector sensors will need to be able to operate at radiation doses of about $10^{15} 1 \mathrm{MeV}$ equivalent neutrons $/ \mathrm{cm}^{2}$. Initial studies of Czochralski and $n$-on- $p$ sensors irradiated up to $4.5 \times 10^{14} 24 \mathrm{GeV}$ protons $/ \mathrm{cm}^{2}$ 
are promising and show that the charge collection efficiencies saturate at acceptable bias voltages [25]. Three-dimensional sensors are another alternative that could be investigated.

Two different vertex-detector geometries are envisaged. One is to shorten the strips, the other is to use pixels. Removing the RF foil that separates the VELO sensors from the primary beampipe vacuum would reduce the radiation length before the first measurement by $3 \%$ and improve the proper time resolution of $B$ meson decays.

A preliminary study uses $B_{s}^{0} \rightarrow D_{s}^{\mp} K^{ \pm}$decays simulated at a luminosity of $6 \times 10^{32} \mathrm{~cm}^{-2} \mathrm{~s}^{-1}$. Events with large numbers of interactions are employed to simulate larger effective luminosities up to $2 \times 10^{33} \mathrm{~cm}^{-2} \mathrm{~s}^{-1}$. In a first step, the existing HLT VELO trigger algorithm is added without modifications into the L0 trigger. We find that the minimum bias rate keeps rising with luminosity and saturates the bandwidth well below our target luminosity. A better approach is to combine the L0 trigger with a detached vertex trigger and to read out at a $40 \mathrm{MHz}$ rate. We require a transverse energy $E_{T}>3 \mathrm{GeV}$ from the hadron trigger and combine this with a matched track that has a transverse momentum $p_{T}>2 \mathrm{GeV} / c$ and an impact parameter $\delta>50 \mu \mathrm{m}$. In this combined trigger the minimum bias rate does not depend strongly on the luminosity and the triggered event yield scales linearly with the luminosity. In addition, the total trigger efficiency is $60 \%$ larger when compared with the existing baseline.

\section{CONCLUSIONS}

We have presented a plan to upgrade the $\mathrm{LHCb}$ experiment after about five years of running. This programme is motivated by new physics beyond the Standard Model that can be studied with a ten times larger data sample. The $\mathrm{LHCb}$ upgrade would allow a precise measurement the weak mixing phase $\phi_{s}$ at its Standard Model value and to probe new physics in $B$ meson decays at an unprecedented level of a few percent or better. Initial estimates show that an excellent science case can be developed. The plan is to upgrade the $\mathrm{LHCb}$ detector such that it can operate at ten times the design luminosity, i.e. at
$\mathcal{L} \sim 2 \times 10^{33} \mathrm{~cm}^{-2} \mathrm{~s}^{-1}$. The upgraded experiment can run with or without an LHC luminosity upgrade. The $\mathrm{LHCb}$ upgrade will require a firstlevel detached vertex trigger. To achieve this, the vertex detector sensors will need to be replaced and many other sub-systems will need to be improved. Feasibility studies for physics and trigger, and $\mathrm{R} \& \mathrm{D}$ for detectors and front-end electronics are now required.

\section{REFERENCES}

1. E. Rodrigues, these proceedings.

2. H. Ruiz, these proceedings.

3. L Garrido, these proceedings.

4. G. Corti, these proceedings.

5. A. Carbone, these proceedings.

6. Y. Xie, these proceedings.

7. P. Robbe, these proceedings.

8. N. Magini, these proceedings.

9. M. Smizanska, these proceedings.

10. S. de Capua, these proceedings.

11. R. Muresan, these proceedings.

12. L. Wolfenstein, Phys. Rev. Lett. 51, (1983) 1945.

13. G. D'Ambrosio et al., Nucl. Phys. B645 (2002) 155.

14. V.M. Abazov et al., hep-ex/0701012

15. L. Fernandez, at Physics at LHC, Cracow, Poland 2006, CERN/LHCB/2006-047 (2006).

16. Z. Ligeti et al., Phys. Rev. Lett. 97 (2006) 101801.

17. Heavy Flavour Averaging Group, E. Barberio et al., hep-ex/0603003, updated (ICHEP06): http://www.slac.stanford.edu/xorg/hfag/

18. M. Raidal, Phys. Rev. Lett. 89 (2002) 231803.

19. R Antunes Nobrega et al., LHCb TDR 9, CERN/LHCC/2003-30 (2003).

20. J. Libby, at CKM 2006, Nagoya (2006).

21. A. Giri et al., Phys. Rev., D68 (2003) 054018.

22. D. Atwood, I. Dunietz and A. Soni, Phys. Rev. Lett. 78 (1997) 3257.

23. M. Gronau, Phys. Lett., B557 (2003) 198.

24. M. Hazumi, presented at Flavour in the LHCEra Workshop, CERN (2005).

25. C. Parkes, Nucl. Inst. and Meth. A569 (2006) 115. 